\title{
Manufacturing and characterization of extracellular vesicles from umbilical cord-derived mesenchymal stromal cells for clinical testing
}

\author{
EVA ROHDE $^{1,2,4}$, KARIN PACHLER $^{1,2,3} \&$ MARIO GIMONA $^{1,2,3,4}$ \\ ${ }^{1}$ GMP Laboratory, Spinal Cord Injury and Tissue Regeneration Center Salzburg (SCI-TReCS), Paracelsus Medical \\ University (PMU), Salzburg, Austria, ${ }^{2}$ Department of Transfusion Medicine, Paracelsus Medical University (PMU), \\ Salzburg, Austria, ${ }^{3}$ Research Program Nanovesicular Therapies, Paracelsus Medical University (PMU), Salzburg, \\ Austria, and ${ }^{4}$ Celericon Therapeutics G.m.b.H., Paracelsus Medical University (PMU), Salzburg, Austria
}

\begin{abstract}
Extracellular vesicles (EVs) derived from mesenchymal stromal cells (MSCs) may deliver therapeutic effects that are comparable to their parental cells. MSC-EVs are promising agents for the treatment of a variety of diseases. To reach the intermediate goal of clinically testing safety and efficacy of EVs, strategies should strive for efficient translation of current EV research. On the basis of our in vitro an in vivo findings regarding the biological actions of EVs and our experience in manufacturing biological stem cell therapeutics for routine use and clinical testing, we discuss strategies of manufacturing and quality control of umbilical cord-derived MSC-EVs. We introduce guidelines of good manufacturing practice and their practicability along the path from the laboratory to the patient. We present aspects of manufacturing and final product quality testing and highlight the principle of "The process is the product." The approach presented in this perspective article may facilitate translational research during the development of complex biological EV-based therapeutics in a very early stage of manufacturing as well as during early clinical safety and proof-of-concept testing.
\end{abstract}

Key Words: clinical testing of extracellular vesicles, exosomes, extracellular vesicles (EVs), extracellular vesicles therapeutics, pharmaceutical manufacturing

\section{Introduction}

Extracellular vesicles (EVs) display numerous biological properties depending on their parental cells [1]. Within the past decade, evidence has accumulated that EVs can mediate therapeutic effects that partially or entirely reflect the biological activity of the cells of origin $[2,3]$. EVs are released into the secretome from potentially all pro- and eukaryotic cell types and can be enriched in a vesicular secretome fraction (VSF) from primary cells, induced pluripotent stem cells (iPSCs), embryonic stem cells or immortalized cell lines using different isolation strategies [4-7]. EVs of variable sizes, such as small EVs of roughly $50-150 \mathrm{~nm}$ in diameter (also referred to as exosomes), and larger microvesicles (up to $1000 \mathrm{~nm}$ ), can be harvested for therapeutic purposes from body fluids or cell culture supernatants. Emerging concepts try to exploit their therapeutic effects in a kind of "next-generation cell therapy" [8]. This cell-free "cell therapy 2.0" can help to circumvent complicated handling issues of biological therapeutics containing viable cells and may efficiently mediate therapeutic activity while avoiding potential harmful side effects of cells. When it comes to clinical testing of EV therapeutics, it is clear that EVs are biological therapeutics, and internationally harmonized regulatory frameworks exist and are applicable [3]. Regulatory aspects impose several challenges on manufacturers, distributors and clinical research teams. The pharmaceutical classification of EV therapeutics as "biological therapeutics" is not merely a semantic specification but defines a precise set of regulatory requirements $[6,9,10]$. Existing in vitro and in vivo experimental evidence shows that EVs from umbilical cord (UC)-derived multipotent

Correspondence: Eva Rohde, MD, Department of Transfusion Medicine, Salzburger Landeskliniken GesmbH, Müllner Hauptstrasse 48, 5020 Salzburg, Austria. E-mail: e.rohde@salk.at 
mesenchymal stromal cells (MSC) promote anti-apoptotic [11,12], pro-angiogenic [13-15] and antifibrotic activities $[16,17]$ and in particular exhibit immunomodulatory effects similar to their source cells [18-28]. Data from various experimental treatments of more than 1900 patients published in more than 90 clinical studies using UC- or Wharton's jelly-derived MSCs argue for a high safety profile of UC-MSC application. With regard to efficacy, in particular, results from randomized placebo controlled phase $1 / 2$, phase 2 and phase 3 studies (30/93) show therapeutic effects in different pathological conditions, including neurological, hematological, immunological, liver, cardiac, endocrine, musculoskeletal, skin, ophthalmological and pulmonary diseases [29]. These observations and the assumption that UC-MSC-EVs may specifically mediate tissue protection and regeneration via immunomodulatory, anti-inflammatory or antifibrotic activity, reflecting the immunologically exceptional circumstances of UC tissue, which enable the organism to tolerate different individual immune systems during pregnancy, prompted us to focus on the therapeutic potential of UC-MSC-EVs. In this perspective, we discuss the manufacturing requirements based on our experience with clinical cell therapy and present the strategy we pursue to generate and characterize UCMSC-EVs for clinical examination.

\section{Principles and strategic decisions in the early developmental stage of manufacturing investigational medicinal products/ investigational new drugs}

\section{The process is the product}

The technical term for a novel, therapeutically active substance that is not yet an approved drug and is going to be tested in nonclinical and clinical studies is "investigational medicinal product" (IMP) in Europe and "investigational new drug" (IND) in the United States. Many efforts during development relate to the search for the active ingredient(s) that mediate a certain biological activity responsible for desired therapeutic but also for undesired adverse effects. This is specifically challenging in the case of biological substances composed of complex macromolecular structures that may induce pleiotropic activities, as it is the case for EVs. Furthermore, innovative manufacturing technologies can go ahead of technological tools for detection or characterization of novel therapeutic agents. For the nanometersized EVs, this means that technological limits may hamper their detailed quantitative and qualitative (physicochemical, immunochemical or functional) characterization. However, there is neither an inevitable necessity to completely characterize or identify the active substance of an IMP/IND, nor is it required to provide a detailed concept about the mode of action (MoA) in the early phase of biopharmaceutical development before the completion of phase 2 clinical trials [30]. This early phase of biological drug development offers the chance for a reasonable and rather practical approach: "The process is the product." Such an approach is common in biopharmaceutical development and suggests that if manufacturing repeatedly adheres to highly standardized procedures, the resulting batch consistency is probably high. To check batch-to-batch consistency, newly generated batches are compared with previous preparations by using biochemical, biophysical and functional assays. This practice, however, does not absolve the need for a continuous search to identify the therapeutic substance(s) mediating a certain therapeutic effect (or effects) via specific MoA in various functional in vitro or in vivo assays. At later developmental stages of clinical testing, all model systems established have to be evaluated with regard to their disease relevance repeatedly and have to be related to clinical observations.

\section{Essential issues to be addressed and decisions to be made}

If manufacturers consequently follow the principle that "the process is the product," and for as long as regulatory authorities accept this strategy, it is obvious that decisions regarding the production process are helpful very early in development. Once decided, production design should remain unaltered, and, if necessary, any changes must be clearly rationalized and any potential new risks thoroughly evaluated. After each modification of the manufacturing process, the circuits of characterization and functional testing with systematic comparison to previously generated batches must start again to confirm that the particular change has no effects on the biological activity and characteristics of the final product. This implies that manufacturers, together with treating physicians, should decide on the disease to be treated and on the therapeutic approach at the earliest possible time. The target disease and its conditions will profoundly influence the manufacturing strategies with regard to treatment options of novel drugs. For example, systemic application will require a substantially higher total dose for each patient compared with clinical indications that allow local application to the site of injury. Disease conditions that require high local or systemic heparin doses may counteract the treatment by preventing EV docking to the membrane of target cells. Conditions that see high and prolonged dosing of antiviral, immunosuppressive or anti-inflammatory medication may likewise change to functional profile of the EV-based therapeutic agent. A specific situation may also arise in multiple 
trauma-associated injuries, such as those of the spinal cord. Lifesaving interventions and medication may compete with EV action and prevent the option for early neuroprotective EV treatment.

Beyond the relevance of the target disease, a non-exhaustive compilation of essential issues and decision-making needs with regard to novel EV therapeutics and its potential clinical use is as follows:

- The therapeutic target for a novel EV-based IMP/ IND

- The proposed therapeutic potency, including the disease relevant in vitro and in vivo models: according to the Code of Federal Regulation (CFR) of the U.S. Food and Drug Administration (FDA), potency is the specific ability or capacity of a product to affect a given result (21CFR600.3s)

- If available, the related MoA affected by a given active drug component(s)/active ingredient(s), resulting in the observed potency

- The planned route of administration (systemic versus local application of the IMP/IND)

- Single versus multiple administration and dose of the IMP/IND per treatment

- Personalized (in the case of autologous cells) versus common (off-the-shelf) use of allogeneic cell-derived EVs

- The source of producer cells (human, mammalian, non-mammalian, etc.)

- Unmodified versus genetically modified producer cells for EV generation

- Naive, naturally or endogenously loaded versus artificially or externally loaded EVs

- Patient numbers intended to be treated-focus on frequent versus rare disease indication

Our approach for clinical examination of UCMSC-EVs is as follows. We intend to exploit the antiinflammatory, anti-scarring, antifibrotic and neuroprotective potential of UC-MSC-EVs. Although there exist several observations of in vitro and in vivo potency, a definite MoA is still unknown, and we can currently only speculate on the nature of active ingredients enriched within the VSF (UC-MSC-EV) fractions. Patients with target diseases who may benefit from UC-MSC-EV therapeutics include those suffering from local inflammatory processes, scar formation and fibrotic alterations or neurodegeneration with circumscribed functional organ damages (e.g., non-union bone fractures, tendon ruptures, neurodegeneration). We will choose local application strategies for UCMSC-EVs, which has direct implications on the number and size of dosages and thus on the batch sizes we envisage for production. We aim to prepare off-theshelf EV therapeutics and will use allogeneic and naive, endogenously generated EVs from unmodified UC-
MSCs for clinical testing. Pathological conditions we are currently focusing on range from common diseases with large patient numbers (e.g., nonunion bone fractures or enthesopathies) to rare neurodegenerative diseases such as traumatic spinal cord injury. All these considerations result in a strategy that enables planning and setting up the steps necessary for a workflow that covers manufacturing, characterization, quality control, preclinical and clinical safety and efficacy testing of UC-MSC-EVs as biological IMPs/INDs.

\section{Manufacturing aspects for EV therapeutics}

Saving cost of goods (CoGs)

On the basis of the aforementioned issues and according to the principle that the "process is the product," we have established upstream and downstream processes in workflow for the generation of UC-MSCEVs with reproducible molecular and biological characteristics (Figure 1). General decisions regarding manufacturing start as early in the process as choosing producer cell lines, media, growth-supporting sera, solid two- or three-dimensional growth support, hypoxic conditions or metabolic preconditioning and then extend to the intended use. A stringent quality control process is required and demands a fair amount of test substance, leading to batch size as a dictating parameter. Consequently, the scalability of cell expansion processes is essential, and considerations about overall CoGs in determining the future product pricing will influence the course of preclinical to clinical development, even at an early developmental stage.

From this perspective, closed manufacturing systems have a clear advantage over standard, open twodimensional plastic surface expansion models. However, quality control of the producer cells within bioreactor systems can be restricted in certain cases. Several manufacturers of EV therapeutics favor bioreactor approaches with the argument that closed systems enable significant scaling down of clean-room requirements. This argument is certainly valid but presents a benefit only when the process of EV enrichment or purification, as well as the steps of filling and finishing the final product, remain in a closed system as well. Currently, this is difficult to achieve for small and medium-sized biotech companies or academic sites working according to Good Manufacturing Practice (GMP).

Another important issue influencing the CoGs is the level of outsourcing capabilities. Manufacturers should carefully determine cost savings and complementary aspects for cell expansion and EV enrichment and balance this with efforts in the selection of a contract manufacturing organization (CMO) and technology transfer. The complications related to satisfactory technology transfer are generally underestimated, 
EV Manufacturing Scheme

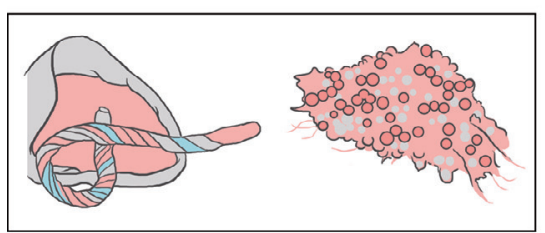

1

Source tissue harvest \& cell isolation

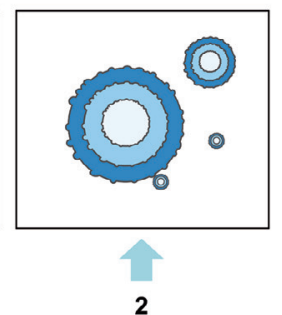

Primary MSC culture

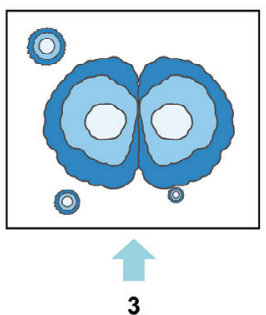

Immortalization

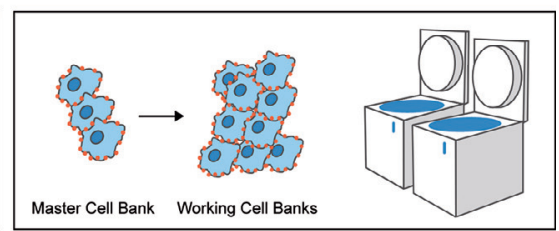

Cell bank manufacturing \& storage
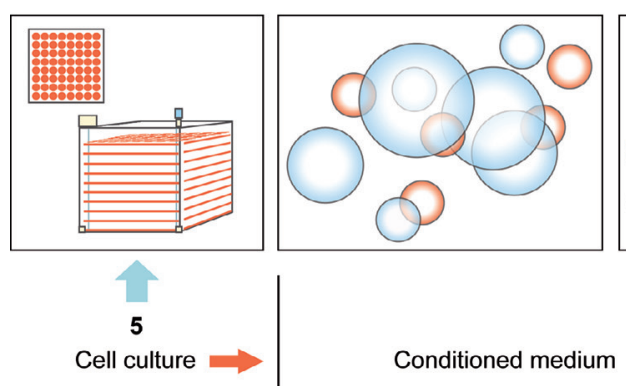

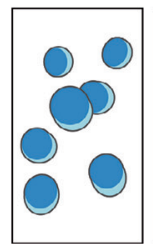

6
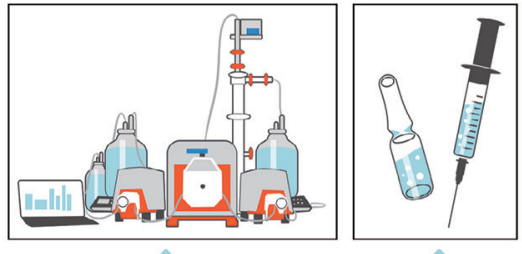

7

EV purification TFF/UC

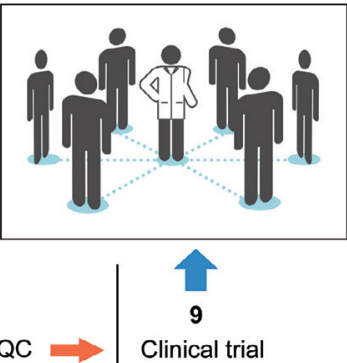

Clinical trial

Principle For Early Stage Biological IMPs/INDs: „THE PROCESS IS THE PRODUCT““

Downstream Processing

CT

Figure 1. Manufacturing of UC-MSC-EV potential therapeutics. The manufacturing scheme of UC-MSC-EV-based putative therapeutics depicts the main steps covering the upstream processing phase and the downstream technologies until filling and storage established in compliance with GMP. Growth-supporting additives such as human- or animal-derived sera should be depleted of endogenous extracellular vesicles before use in cell expansion to prevent contamination of the source cell-derived EVs, which are isolated from conditioned medium. EV purification is performed using tangential flow filtration (TFF) combined with a short ultracentrifugation (UC) step. A consistent quality control (QC) strategy for the final product testing is needed to fulfill regulatory requirements and to achieve the approval for clinical testing of the putative novel drug. In a very early stage of clinical testing of biological investigational medicinal products (IMPs)/investigational new drugs (INDs), the principle of "the process is the product" can be applied. It helps to bridge the initial phase of lacking knowledge regarding the active substance and the MoA responsible for a specific therapeutic activity until proof of mechanisms and therapeutic activity are identified and a clinical trial (CT) initiated.

leading to unexpected delays and a sharp increase in costs in the product development phase. Moreover, even if the technical competence of a CMO has been approved by the client and all legal/contractual issues have been resolved, few contract manufacturers hold an appropriate manufacturing license for EVs at the time a contract is signed. Process establishment and the mandatory process validation inevitably delay the start of the manufacturing process and hence extend the time to market (or clinical trial). In addition, few EV therapeutic developers can transfer robust standard operating procedure (SOP)-guided processes at an early stage, and on average, a substantial amount of process development and SOP amendment is required before a stable process is fully implemented at a new manufacturing site.

To a large degree, such considerations extend to approval of the therapeutic substance and marketing authorization after the successful demonstration of efficacy in a pivotal phase 3 clinical trial. Once again, steps must be considered early and carefully for both academic and small and medium-sized enterprises because the financial requirements for phase 2 and phase 3 clinical trials are considerably high due to manufacturing costs, but particularly due to costs related to clinical testing.

\section{Scalability of the manufacturing train}

Whenever a manufacturing process is broken down into the various stages and examined in light of the mandatory volume of the biopharmaceutical, the requirement for developing practical procedures becomes evident. Pragmatic, step-by-step assembly will reveal bottlenecks and high-risk elements in the process design that must be solved while maintaining overall GMP compliance of the entire workflow. For an affordable pharmaceutical manufacturing strategy, 
the use of small-volume bioreactors may suffice until the early clinical phase testing. If the requirements exceed a level of several hundred doses, manufacturers may reconsider the suitability of this approach. In any case, it is important to develop a strategy with a clear view on the required amount and the achievable batch size for each manufacturing run. Thus, dose considerations based on preclinical proof-of-concept and dose-response studies as well as the establishment of primary versus immortalized master and working cell banks profoundly influence the scale and layout of the upstream processing [6].

For both primary and permanent cell lines, a thorough, risk-based analysis must be conducted, and the arising safety concerns differ considerably between the two. Overall virus safety must be determined (details of risks are discussed subsequently) and for cells carrying a transgene, the requirements are more stringent. Although the absence of the transgene in the EV product can be monitored, genetic modification of primary cells will nevertheless require an extended in vitro and in vivo testing. A worthwhile approach is the comparison (by proteomic and nucleic acid profiling) of EVs derived from the immortalized cell source versus the parental cell before transformation. Although the gene expression profile can be altered substantially after transformation, these changes may not become manifested in the secreted EVs. However, this must be verified on a case-by-case basis. For our specific situation, we have decided to use hTERT instead of c-myc for immortalization and have observed a rather stable gene expression profile for the relevant surface markers.

Additional scalability issues relate to downstream processing and initial enrichment of EVs from large volumes of conditioned media. Precipitation protocols have not improved EV purification, and a final concentration to suitable volumes can still be achieved via ultracentrifugation. An attractive alternative for the initial enrichment of vesicles is serial filtration based on the technology of tangential flow filtration (TFF), which has been used for virus isolation for some time $[31,32]$. We have discussed the opportunities of TFF as a convenient EV enrichment method in a previous article [6]. TFF combined with a final ultracentrifugation step is an efficient, scalable and rapid method in our hands.

\section{Preclinical and clinical product characterization}

In early-stage trials (mostly phase 1 and $2 a$ ), the primary goal of the clinical evaluation of EV-therapeutics is the safety of the recipient (volunteers or patients). This requires extensive product testing to address any possible safety concerns. From a strict manufacturing perspective, the definition of the active compound or the MoA is not of principal importance. In the absence of a known MoA, the batch consistency and reproducibility of the manufacturing process are prime parameters for every biopharmaceutical. Particularly for UC-MSCEV-therapeutics, the current process still defines the product, and therefore product characterization via a series of surrogate markers helps provide sufficient data to meet regulatory requirements (discussed subsequently). It is also necessary to include stability programs to determine the shelf life of EV therapeutics. Data supporting the definition of the shelf life will in turn not only influence the frequency and amount of the manufacturing runs but also provide necessary information for subsequent clinical trials. Definition of acceptance and release (as well as rejection) that focus particularly on the characterization of the active drug component(s) and planning and validation of suitable quality control measures go hand in hand with the validation of the manufacturing process. In light of required GMP compliance, these criteria, measures and validation strategies are a part of the developing manufacturing process, which may undergo several changes over the time. Eventually, after all milestones have been reached and significant efficacy has been shown in pivotal phase III clinical trials, the EV-based therapeutic may get a changed status from an EV-based biological IMP/IND to a novel drug ready for approval for marketing authorization.

\section{Potential risks associated with EV manufacturing}

Apart from any purity and identity issues of EV-based products, a primary concern is obviously the high congruence between small EVs (such as exosomes) and virus. Current enrichment and purification strategies have their origin in virus and virus-like particle manufacturing (e.g., tangential flow filtration, ultracentrifugation, polyethylene glycol precipitation, serial filtration, size exclusion chromatography, a.s.o.). Thus, any virus that is present in the conditioned medium will become enriched in the final product and thereby potentially increases the risk profile. Chemical virus inactivation seems as inappropriate as radiation: in both cases, the final product will be altered, and the impact of radiation on product performance cannot be determined a priori. For EVs that are used as sole carriers and transport vehicles of exogenously loaded compounds and substances (particularly RNA-based formulations), excessive radiation may in fact be counterproductive and could cause severe complications due to structural alterations or increased instability of the nucleic acid chain.

Current stringent virus testing includes both in vitro and in vivo animal testing but can only focus on known pathogenic strains. There are initiatives to rewrite specific International Council for Harmonisation (ICH) guidelines to remove the requirement for in vivo testing 
with the goal of reducing the number of animals used in pharmaceutical testing in general. Some in vivo testing may be replaced by next-generation sequencing, which may also enable the search for as-yet-unknown or underestimated virus of significance for human pathology. It seems that at present, a residual risk for virus contamination remains associated with all natural EV-based, cell-derived products. However, the risk does not appear increased compared with other allogeneic stem cell therapy approaches that use viable nonirradiated cells as the active substance.

Monitoring of endotoxin levels appears relevant given that many high-throughput filter technologies may result in the excessive production of pyrogenic particles or substances. Stainless steel connections can help to reduce the generation of small particles but will not eliminate the need for close endotoxin monitoring in the final product.

Manufacturers are in any case required to provide a thorough, risk-based analysis of the entire manufacturing process and can thus analyze the specific risks associated with their proprietary manufacturing procedure.

\section{Final product quality control and standardized characterization}

\section{Principles of pharmaceutical quality control}

Equally important to a well-controlled and scalable manufacturing process is the subsequent adequate characterization of the final product. The requirements of quality control testing for biopharmaceuticals are described in detail in the European Medicines Agency's "Guideline on the Requirements for Quality Documentation Concerning Biological Investigational Medicinal Products in Clinical Trials" and include the principles of the identity, purity, impurity, potency, safety and stability determination that are also valid for potential EV therapeutics [33]. These principles are elaborated considering the specific situation that the therapeutically active substance(s) or active ingredient(s) in biopharmaceuticals cannot easily be defined at an early time point in the course of development. "Acceptance criteria for IMP quality attributes should take into account safety considerations and the stage of development. Since acceptance criteria are normally based on a limited number of developmental batches and batches used in nonclinical and clinical studies, their nature is inherently preliminary" [33]. Nevertheless, manufacturers are expected to collect and provide sufficient data for the authorities to evaluate a therapeutic activity/potential and the safety profile of the IND/IMP to justify clinical evaluation in early developmental stages. Characterization of a putative biological drug substance includes the determination of physicochemical properties, biological activity, immunochemical properties, purity and impurities by appropriate techniques. Acceptance criteria can be established and justified based on data obtained from lots used in preclinical and/or clinical studies, data from lots used for demonstration of manufacturing consistency and data from stability studies. If needed, manufacturers establish appropriately characterized in-house reference materials, which serve for biological and physicochemical testing of produced batches. New analytical technologies and modifications to existing technology are continually being developed and should be evaluated in adherence to the ICH guideline "Test Procedures and Acceptance Criteria for Biotechnological/ Biological Products" [34]. Technologies, including biophysical, biochemical and immunochemical methods, should help characterize substances of interest and reveal the therapeutically active drug component.

\section{Quality control and release criteria of VSF UC-MSC- EVs as a final product}

The approach applied in our manufacturing laboratory using a multimodal matrix for release testing of UCMSC-EVs in the VSF is presented in Table 1. Parameters indicating values, ranges or marker profiles to determine parental cell characteristics and identity, purity and impurities of the EV products as well as related test methods that have been chosen on the basis of our experience from UC-MSC-EV engineering runs. For the aim of providing UC-MSC-EVs as a biological for clinical testing, the quality control strategy starts with the determination of the amount and viability of the parental cells. The number of viable cells at time of harvest in a defined volume of conditioned medium can be used to express the EV quantity in cell equivalents (CE). Although it is a relative quantification method, the CE may be helpful for comparative testing of therapeutic potency in dose-finding studies. Another minimum criterion is the identification of the parental cell phenotype by flow cytometry analysis, resulting in a defined surface marker profile. Although it must be acknowledged that MSCs are a heterogeneous population of cells, current guidelines (such as the standard International Society for Cellular Therapy criteria) should be followed as a minimal criteria catalog must be established [35]. Values can be adjusted depending on the source and nature of the cells (primary or immortalized), but overall a minimum MSC profile should be established. As discussed in the preceding section on manufacturing, manufacturers of therapeutic EVs should consider the level of purity that can be achieved and a level that is accepted for the final product.

Definitions of purity do not necessarily allude to a semantic (i.e., do we speak about vesicle or non-vesicle 
Table I. Multimodal Testing of Quality Control Parameters of UC-MSC EVs in the Vesicular Secretome Fraction.

\begin{tabular}{|c|c|c|}
\hline Parameter & Release criteria (range or profiles) & Method \\
\hline \multicolumn{3}{|l|}{ Parental cells } \\
\hline Cell count and viability & $\geq 90 \%$ viable cells, cell count determines cell equivalent & Manual count, trypan blue method \\
\hline \multirow[t]{2}{*}{ Cell surface marker profile } & $\begin{array}{l}\geq 95 \% \mathrm{CD} 29^{+}, \mathrm{CD} 44^{+}, \mathrm{CD} 73^{+}, \mathrm{CD} 90^{+}, \mathrm{CD} 105^{+}, \\
\mathrm{CD} 166^{+}\end{array}$ & Multi-color flow cytometry \\
\hline & $\leq 2 \% \mathrm{CD} 14^{-}, \mathrm{CD} 19^{-}, \mathrm{CD} 34^{-}, \mathrm{CD} 45^{-}, \mathrm{MHC}$ class $\mathrm{II}^{-}$ & \\
\hline \multicolumn{3}{|l|}{ Identity purity and impurities } \\
\hline EV quantity & $4-8 \times 10 \mathrm{E}^{11} / \mathrm{mL}$ & NTA \\
\hline EV size & $80-120 \mathrm{~nm}$ & NTA \\
\hline EV particle identity & Percentage of CD63+, CD81+, CD73+ $(\geq 10-15 \%)$ & Fluorescent NTA \\
\hline \multirow[t]{3}{*}{ EV Surface Marker Profile } & $\mathrm{CD} 3^{+}, \mathrm{CD} 81^{+}, \mathrm{Tsg} 101^{+}$ & Western blotting \\
\hline & $\begin{array}{l}\mathrm{CD}^{+}, \mathrm{CD} 29^{+}, \mathrm{CD} 44^{+}, \mathrm{CD} 49 \mathrm{e}^{+}, \mathrm{CD} 63^{+}, \mathrm{CD} 81^{+}, \\
\mathrm{CD}^{+} 3^{+}, \mathrm{CD} 105^{+}, \mathrm{MCSP}^{+}\end{array}$ & $\begin{array}{l}\text { Flow cytometry based bead array MACS } \\
\text { Plex }\end{array}$ \\
\hline & $\begin{array}{l}\mathrm{CD} 14^{-}, \mathrm{CD} 19^{-}, \mathrm{CD} 34^{-}, \mathrm{CD} 45^{-}, \mathrm{CD} 142^{-}, \mathrm{MHC} \\
\text { class } \mathrm{I}^{-}, \text {class } \mathrm{II}^{-}\end{array}$ & \\
\hline \multicolumn{3}{|l|}{ Microbial impurities } \\
\hline EV endotoxin & $\leq 5 \mathrm{EU} / \mathrm{kg}$ bodyweight of the recipient & Tests according to Pharm. Eur. (2.6.14) \\
\hline EV sterility & Negative & Tests according to Pharm. Eur. (2.6.1) \\
\hline EV mycoplasma & Negative & Tests according to Pharm. Eur. (2.6.7) \\
\hline \multicolumn{3}{|l|}{ Informative testing ${ }^{\mathrm{a}}$} \\
\hline EV protein concentration & $\mu \mathrm{g} / \mathrm{mL}$ or $\mu \mathrm{g} /$ particle & QuBit3 Fluorimetric Assay \\
\hline EV miRNA/RNA profile & $\begin{array}{l}\text { Absence of inhibitory or problematic (e.g., mt or } \\
\text { tumorigenic) miRNAs }\end{array}$ & $\begin{array}{l}\text { AGILENT Bioanalyzer profiling, } \\
\text { polymerase chain reaction or deep } \\
\text { sequencing }\end{array}$ \\
\hline EV cytokine profile & Provide data on absence of pro-inflammatory cytokines & Multiplex assay \\
\hline Enhanced cell proliferation & & xCELLigence In Vitro Cell Assay \\
\hline Inhibition of T-cell crowth & & In vitro potency assay \\
\hline Neuroprotective activity & & In vitro/in vivo potency assays \\
\hline Antifibrotic activity & & In vitro/in vivo potency assays \\
\hline Anti-inflammatory activity & & In vitro/in vivo potency assays \\
\hline Accelerated bone healing & & In vitro/in vivo potency assays \\
\hline
\end{tabular}

Multimodal matrix testing of UC-MSC EVs in the VSF. Parental cell characterization, identity, purity and impurity determination of EV preparations is performed for the standard quality release testing of all research scale preparations and for GMP training and GMP clinical runs. Additive informative testing is performed on a non-regular basis for extensive characterization but is not part of the pharmaceutical quality release procedure. These assays provide important supportive data about protein, RNA and cytokine profiles and about functional effects of UC-MSC-EVs.

${ }^{a}$ The categories in this section are not part of release testing but provide additional information.

preparations?) but rather to a regulatory topic. To qualify for using the term "vesicles" and discriminate such a product from a secretome, a product composed of "UC-MCS-EVs" should contain vesicles as the major particulate species. The differences in the views on purity and identity become most obvious when UC-MCS-EV preparations are characterized by fluorescent nanoparticle tracking analysis (NTA) or electron microscopy (the latter not being a release test). At this point, a clear view on the underlying principles and basic physics seems appropriate. It is a common approach to determine the number of EVs by NTA. It must be acknowledged, however, that the results from NTA can only be used to indicate the total amount of particles in a solution and do not a priori determine the number of membrane-surrounded vesicles.

This situation may require reconsideration of the current approach to accept the ratio of total particles (by NTA) and total protein content as a level of purity. If, however, this strategy can consider the amount of CD9/CD63/CD81-positive particles in relation to the total protein content, the result may be more straightforward. Nevertheless, it should be clearly demonstrated that the presence of certain proteins is unfavorable for the biological activity of the EV product. In addition, highly purified and protein-free EV preparations seem to be highly unstable in storage, and long-term preservation of functionally competent EVs seems to require the addition of exogenous proteins like albumin. Whether the ratio between particles (and measured by NTA) and protein concentration can be used as a reliable indicator for purity as proposed by others [36] has to be confirmed.

Indeed, recent developments in the field have addressed this issue and the dual comparative measurement of total particles versus particles positive for standard vesicle surface markers (CD9, CD63, CD81) by fluorescent NTA reveal that most preparations may contain as little as $5-10 \%$ of the total particle mass as EVs. Thus, manufacturers and sponsors of clinical trials testing MSC-derived EV preparations may instead consider the product under scrutiny a 
vesicle-enriched secretome fraction (or VSF) than a pure or at least highly enriched $(>70 \%)$ vesicle product [6]. The newly designed ZetaView DL-NTA Dual Laser from ParticleMetrix acknowledges these considerations and may assist manufacturers and researchers in defining their particle solutions more precisely. The concomitantly determined size distribution of the particles cannot be used to insinuate any functionality of the manufactured product at present. There exists no scientific standard clearly attributing a certain function to vesicles of a particular size even within the range of 50-200 nm. Furthermore, the various methods used to define particle size do not result in consistent data because even similar strategies (e.g., NTA) can result in differences of $15-50 \%$ in the determined size.

Finally, a rather simple flow cytometry-based surface marker analysis of the EV preparation can be used to control the manufacturing process and to obtain a profile confirming the identity of the product. A novel human MACSPlex Exosome Kit (Miltenyi Biotec) is a rapid and reliable test that can be a part of the test matrix and that provides valuable batch consistency information on the product. A systematic evaluation of this multiplex bead-based flow cytometry assay shows that this system allows for a robust, sensitive and reproducible detection of EV surface marker profiles in various EV sample types [37].

Standard safety tests for biological products according to the European Pharmacopoeia include determination of endotoxin levels, sterility and absence of mycoplasma in the final product. These tests serve to exclude microbial impurities and are services provided by specifically certified laboratories. The presented multilayered test matrix allows tight quality control of the manufacturing, packaging and storage process and provides a considerable amount of data regarding the putative novel drug. The compilation of these acceptance and release criteria is a key part of the application documents for regulatory authorities to grant approval for clinical testing of a biological IMP/IND.

\section{Additional information about biological product characteristics}

Further informative testing, such as protein quantification, RNA or cytokine profiling, and functional in vitro and in vivo potency assays are currently not performed within our regular release testing program but can provide valuable additional information that may be used to support a proposed MoA (Table I). In several cases, the lack of precision in particle determination has led researchers and manufacturers to employ total protein mass/protein quantification as a criterion to attempt a preliminary dose determination. Obviously, such a strategy is acceptable for EV harvested cells grown in a serum-free or chemically defined medium but may present limitations with other manufacturing strategies, including serum-supplemented media, coated hollow fiber or stirred tank bioreactors. For the monitoring of a manufacturing process, the information on protein concentration helps to confirm the stability and reproducibility of the process but is not useful as a stand-alone measure for dose finding. To obtain supporting information in addition to the protein mass data, total RNA and micro (mi)RNA determination may be employed. miRNA profiling may help determine the amount of miRNA that is presumably responsible for an intended therapeutic effect [38], such as the antiviral activity of let7f, miR-145, miR-199a and miR-221 [39] or miR-124 to increase chemosensitivity of glioblastoma cells [40]. In addition, it may be used to confirm the absence of undesirable miRNAs - for example, miR-410, which has recently been reported to promote carcinoma cell growth [41]. The AGILENT 2100 Bioanalyzer system provides a simple cartridge-based onchip electrophoresis device that can generate results in reliable quality and provides objective information regarding concentration, size and integrity of RNA, DNA and protein analysis. Moreover, the AGILENT electronic documentation system complies with FDA criteria (21 CFR Part 11) under which electronic records and electronic signatures are regarded to be trustworthy, reliable and generally equivalent to paper records and handwritten signatures [42].

Relevant in vitro and in vivo potency assays may be included depending on the target disease and the proposed MoA. If available, such relevant potency assays should be validated according to GMP guidelines and represent an important part of the specifications of biological IMPs/INDs. In vitro cell-based assays to measure specific biological activity may provide additional useful information, such as stimulation of proliferation or specific cell survival tests. Others can be used to monitor the suppressive capacity of MSC-EVs to inhibit T-cell proliferation [25]. Disease-relevant in vivo animal models should be carefully chosen to provide additional information. For certain human diseases, a suitable animal model may not be readily available. The biological activity of manufactured EV preparations can be tested routinely in a combination of in vivo and in vitro studies to document the intended therapeutic potential. Considerations regarding the putative MoA to address the issue of a proposed therapeutic potency must be based on solid data on the bioavailability of the IMP/IND in the treated organ to calculate reasonable dose sizes. Challenges of robust and predictive potency assays, MoA and proof-ofconcept studies are addressed in detail in one of our previous articles [6]. Of note, there is a clear indication to provide potency data as a preparation for application 
for market authorization (see the ICH guidelines Q6B on test procedures and acceptance criteria for biotechnological/biological products) [34]. For the scope of this article, however, we have focused on the path from the point of clinical-grade manufacturing to clinical testing in humans. For a more market-oriented opinion, the reader is referred to Colao et al. [43].

\section{Characterization of UC-MSC-EVs beyond rapid-release testing}

The analysis of VSF fractions derived from UCMSCs by using methods that are not suitable for rapid release testing may be helpful for additional product characterization and for the search for biological mechanisms responsible for an observed therapeutic potency. Such methods may include (cryo)-electron microscopy, tunable resistive pulse sensing and conventional atomic force microscopy (AFM) and fast scanning liquid AFM, combined with fluorescence detection [44]. In vivo potency testing of the neuroprotective, antifibrotic and antiinflammatory activity and the potential for accelerated bone healing has been investigated in our GMP laboratory and in collaboration with partners involved in basic research, revealing preliminary promising information about the therapeutic potential of UC-MSC-EVs (data not shown).

\section{Discussion}

The observed physiological functions of EVs enriched from various human and non-human cell types have inspired basic science and clinical researchers to investigate the therapeutic potential of putative EV therapeutics in a multitude of model systems [1]. Although there remain more questions than answers regarding EV biology, initial clinical trials investigating EV therapeutics have begun or already been published [45-49]. These early clinical studies reveal an overall low toxicity and a rather constant, at least immediate, recovery of EVs in the circulation after systemic administration. Currently, various safety and pharmacological issues such as biodistribution, bioavailability, pharmacokinetics and pharmacodynamics remain unclear and must be addressed in future preclinical and clinical studies. Our research is currently focusing on the anti-inflammatory, anti-scarring, antifibrotic and neuroprotective potential of UC-MSC-EVs, although the active drug substance has yet to be identified. Other researchers are attempting to exploit the antiviral capacity of UC-MSC-EVs mediated by let7f, miR-145, miR-199a and miR-221 or the ability to increase chemosensitivity via miR-124 transfer to glioblastoma cells exerted by EVs enriched from
Wharton's jelly MSCs $[39,40]$. In addition to the intended therapeutic effects, the risk for detrimental acute or chronic side effects due to unknown biological activity, immunological reactions or overdosing of UC-MSC-EVs is of interest. A recent study reported a strong increase of lung adenocarcinoma growth in a xenograft tumor-model after co-implantation of UCMSCs or UC-MSC-EVs being induced by miR-410, which could be abrogated by siR- 410 . The suggested MoA provided is the regulation of PTEN expression at the post-transcriptional level in lung adenocarcinoma cells [41].

With regard to miRNA levels of EV therapeutics, it may therefore be meaningful to collect informative data while performing release testing that relates to components of the UC-MSC-EV preparations that should not be enriched during manufacturing (Table I). Tumorigenic effects of UC-MSC-EVs may not always be related to the biological activity of miRNAs. As observed in two independent studies, the presence of UC-MSC-EVs either increased drug resistance by enhanced expression of multidrug resistance proteins in vitro in gastric cancer cells or it conferred stemness. However, in both cases, these observations could not be attributed to the presence of specific miRNAs [50,51].

Currently, only a few projects with EV therapeutics have evolved to a state that is mature enough for clinical evaluation. One of the more advanced projects that may soon be entering clinical testing uses engineered bone marrow-derived MSC-EVs with the ability to target oncogenic KrasG12D for the treatment of pancreatic cancer. Significantly, Mendt et al. presented a GMP-compliant manufacturing strategy, including the relevant quality controls and stability testing of their EV-based biological IMP/IND [52]. The bioreactor-based, large-scale production of clinical grade "iExosomes" is combined with an external siRNA loading strategy. Published results argue for a suppression of patient-derived pancreas ductal adenocarcinoma growth in vitro and in vivo and demonstrate a significantly enhanced survival after repetitive systemic treatments in the reported xenograft tumor model. Furthermore, they show validated efficacy data of GMP-grade iExosomes in vitro and in vivo, provide a proposed MoA responsible for the observed in vivo potency and show biodistribution data. The authors argue that enhanced retention of iExos in the circulation is due to CD47-mediated protection of these EVs from intake by monocytes and macrophages [53]. Whether this novel approach against pancreatic cancer, a disease in urgent need for effective new therapies, represents a safe and effective therapy remains to be demonstrated in clinical trials starting in the near future. 


\section{Conclusion}

Here, we intended to shed light on recent developments in the field and raise both the interest in and awareness of the process of pharmaceutical manufacturing of cellbased EV-therapeutics in general and of UC-MSC-EV therapeutics in particular. We highlighted that the "the process is the product" principle is useful for translational research during development of complex biological IMPs/INDS in a very early stage of manufacturing and even during early clinical safety and proof-ofconcept testing. This strategy helps bridge the initial gaps in knowledge regarding the active substance and mechanism or MoA responsible for a certain therapeutic activity until proof of mechanisms and therapeutic activity are identified.

The major quality considerations for biological pharmaceuticals refer to identity, purity, impurities, potency, safety and stability of EV therapeutics on their way from IMPs/INDs to eventually approved therapeutics. Conclusive data regarding the shelf life and overall product stability, the general toxicity and pharmacodynamics relate to safety aspects just as much as do considerations regarding the proposed MoA, the route of administration and dosing. The aspect of purity and knowledge and control of any co-purifying components and excipients will make the manufacturers realize that EV therapeutics may be closer to difficult-tocharacterize secretomes than are purified EV preparations. Quality control should be tackled at multiple levels and include criteria for the producer cells and the resulting EV therapeutics. A consistent control strategy defines release and rejection criteria and embraces the manufacturing process just as much as the subsequent fill and finish steps, the monitoring of storage conditions and the control of distribution paths in a regulatoryand GMP-compliant manner.

We are confident that if the critical points that we have raised in this perspective article are tackled in a structured and focused manner by manufacturers of EV therapeutics, the translation from experimental research to stringently executed and documented clinical testing of MSC-EVs will become a less troublesome and eventually rewarding endeavor.

\section{Acknowledgments}

The authors acknowledge the financial support from Exothera (funded by the European Regional Development Fund and Interreg V-A Italia-Austria 20142020) and WISS2015 "ExtraNeu" (funded by the government of Salzburg State).

Disclosure of interest: ER is chief medical officer and MG is chief scientific officer and a member of the board of directors of Celericon Therapeutics. The authors report no additional commercial, proprietary or financial interest in the products or companies described in this article.

\section{References}

[1] Yanez-Mo M, Siljander PR, Andreu Z, Zavec AB, Borras FE, Buzas EI, et al. Biological properties of extracellular vesicles and their physiological functions. J Extracell Vesicles 2015;4:27066. https://doi.org/10.3402/jev.v4.27066.

[2] Valadi H, Ekstrom K, Bossios A, Sjostrand M, Lee JJ. and Lotvall JO Exosome-mediated transfer of mRNAs and microRNAs is a novel mechanism of genetic exchange between cells. Nature cell biology 2007;9:654-9. https://doi. org/10.1038/ncb1596.

[3] Lener T, Gimona M, Aigner L, Borger V, Buzas E, Camussi $\mathrm{G}$, et al. Applying extracellular vesicles based therapeutics in clinical trials - an ISEV position paper. J Extracell Vesicles 2015;4:30087. https://doi.org/10.3402/jev.v4.30087.

[4] Thery C, Amigorena S, Raposo G, Clayton A. Isolation and characterization of exosomes from cell culture supernatants and biological fluids. Curr Protoc Cell Biol 2006; Chapter 3: Unit 3 22. https://doi.org/10.1002/0471143030.cb0322s30.

[5] Witwer KW, Buzas EI, Bemis LT, Bora A, Lasser C, Lotvall J, et al. Standardization of sample collection, isolation and analysis methods in extracellular vesicle research. J Extracell Vesicles 2013;2. https://doi.org/10.3402/jev.v2i0.20360.

[6] Gimona M, Pachler K, Laner-Plamberger S, Schallmoser K, Rohde E. Manufacturing of Human Extracellular VesicleBased Therapeutics for Clinical Use. Int J Mol Sci 2017;18. https://doi.org/10.3390/ijms18061190.

[7] Nawaz M, Fatima F, Vallabhaneni KC, Penfornis P, Valadi H, Ekstrom K, et al. Extracellular Vesicles: Evolving Factors in Stem Cell Biology. Stem Cells Int 2016;2016:1073140. https://oi.org/10.1155/2016/1073140.

[8] Reiner AT, Witwer KW, van Balkom BWM, de Beer J, Brodie C, Corteling RL, et al. Concise review: developing bestpractice models for the therapeutic use of extracellular vesicles. Stem Cells Transl Med 2017;6:1730-9. https://doi. org/10.1002/sctm.17-0055.

[9] Ilic N, Savic S, Siegel E, Atkinson K, Tasic L. Examination of the regulatory frameworks applicable to biologic drugs (including stem cells and their progeny) in Europe, the U.S., and Australia: part I-a method of manual documentary analysis. Stem Cells Transl Med 2012;1:898-908. https:// doi.org/10.5966/sctm.2012-0037.

[10] Ilic N, Savic S, Siegel E, Atkinson K, Tasic L. Examination of the regulatory frameworks applicable to biologic drugs (including stem cells and their progeny) in Europe, the U.S., and Australia: part II-a method of software documentary analysis. Stem Cells Transl Med 2012;1:909-20. https://doi. org/10.5966/sctm.2012-0038.

[11] Sun L, Li D, Song K, Wei J, Yao S, Li Z, et al. Exosomes derived from human umbilical cord mesenchymal stem cells protect against cisplatin-induced ovarian granulosa cell stress and apoptosis in vitro. Sci Rep 2017;7:2552. https://oi.org/ 10.1038/s41598-017-02786-x.

[12] Joerger-Messerli MS, Oppliger B, Spinelli M, Thomi G, di Salvo I, Schneider P, et al. Extracellular vesicles derived from Wharton's jelly mesenchymal stem cells prevent and resolve programmed cell death mediated by perinatal hypoxia-ischemia in neuronal cells. Cell Transplant 2018;27:168-80. https://doi. org/10.1177/0963689717738256. 
[13] Zhang B, Wu X, Zhang X, Sun Y, Yan Y, Shi H, et al. Human umbilical cord mesenchymal stem cell exosomes enhance angiogenesis through the Wnt4/beta-catenin pathway. Stem Cells Transl Med 2015;4:513-22. https://doi.org/ 10.5966/sctm.2014-0267.

[14] Zhao Y, Sun X, Cao W, Ma J, Sun L, Qian H, et al. Exosomes derived from human umbilical cord mesenchymal stem cells relieve acute myocardial ischemic injury. Stem Cells Int 2015;2015:761643. https://doi.org/10.1155/2015/761643.

[15] Ma J, Zhao Y, Sun L, Sun X, Zhao X, Sun X, et al. Exosomes derived from Akt-modified human umbilical cord mesenchymal stem cells improve cardiac regeneration and promote angiogenesis via activating platelet-derived growth factor D. Stem Cells Transl Med 2017;6:51-9. https://doi. org $/ 10.5966 / \mathrm{sctm} .2016-0038$.

[16] Li T, Yan Y, Wang B, Qian H, Zhang X, Shen L, et al. Exosomes derived from human umbilical cord mesenchymal stem cells alleviate liver fibrosis. Stem Cells Dev 2013;22:845-54. https://doi.org/10.1089/scd.2012.0395.

[17] Fang S, Xu C, Zhang Y, Xue C, Yang C, Bi H, et al. Umbilical cord-derived mesenchymal stem cell-derived exosomal microRNAs suppress myofibroblast differentiation by inhibiting the transforming growth factor-beta/SMAD2 pathway during wound healing. Stem Cells Transl Med 2016;5:1425-39. https://doi.org/10.5966/sctm.2015-0367.

[18] Lee C, Mitsialis SA, Aslam M, Vitali SH, Vergadi E, Konstantinou G, et al. Exosomes mediate the cytoprotective action of mesenchymal stromal cells on hypoxia-induced pulmonary hypertension. Circulation 2012;126:2601-11. https://doi.org/ 10.1161/circulationaha.112.114173.

[19] Ti D, Hao H, Tong C, Liu J, Dong L, Zheng J, et al. LPSpreconditioned mesenchymal stromal cells modify macrophage polarization for resolution of chronic inflammation via exosome-shuttled let-7b. J Transl Med 2015;13:308. https:// doi.org/10.1186/s12967-015-0642-6.

[20] Li X, Liu L, Yang J, Yu Y, Chai J, Wang L, et al. Exosome derived from human umbilical cord mesenchymal stem cell mediates MiR-181c attenuating burn-induced excessive inflammation. EBioMedicine 2016;8:72-82. https://doi.org/ 10.1016/j.ebiom.2016.04.030.

[21] Yu B, Shao H, Su C, Jiang Y, Chen X, Bai L, et al. Exosomes derived from MSCs ameliorate retinal laser injury partially by inhibition of MCP-1. Sci Rep 2016;6:34562. https://doi.org/ 10.1038/srep34562.

[22] Kim DK, Nishida H, An SY, Shetty AK, Bartosh TJ, Prockop DJ. Chromatographically isolated CD63+CD81+ extracellular vesicles from mesenchymal stromal cells rescue cognitive impairments after TBI. Proc Natl Acad Sci U S A 2016;113:170-5. https://doi.org/10.1073/pnas.1522297113.

[23] Mao F, Wu Y, Tang X, Kang J, Zhang B, Yan Y, et al. Exosomes derived from human umbilical cord mesenchymal stem cells relieve inflammatory bowel disease in mice. BioMed research international 2017;2017:5356760. https:// doi.org/10.1155/2017/5356760.

[24] Monguio-Tortajada M, Roura S, Galvez-Monton C, Pujal JM, Aran G, Sanjurjo L, et al. Nanosized UCMSC-derived extracellular vesicles but not conditioned medium exclusively inhibit the inflammatory response of stimulated $\mathrm{T}$ cells: implications for nanomedicine. Theranostics 2017;7:270-84. https://doi.org/10.7150/thno.16154.

[25] Pachler K, Ketterl N, Desgeorges A, Dunai ZA, LanerPlamberger S, Streif D, et al. An in vitro potency assay for monitoring the immunomodulatory potential of stromal cell-derived extracellular vesicles. Int J Mol Sci 2017;18. https://doi.org/10.3390/ijms18071413.

[26] Bai L, Shao H, Wang H, Zhang Z, Su C, Dong L, et al. Effects of mesenchymal stem cell-derived exosomes on experimental autoimmune uveitis. Sci Rep 2017;7:4323. https://doi.org/10.1038/s41598-017-04559-y.

[27] Song Y, Dou H, Li X, Zhao X, Li Y, Liu D, et al. Exosomal miR-146a contributes to the enhanced therapeutic efficacy of interleukin-1beta-primed mesenchymal stem cells against sepsis. Stem Cells 2017;35:1208-21. https://doi.org/10.1002/stem.2564.

[28] Zhang Q, Fu L, Liang Y, Guo Z, Wang L, Ma C, et al. Exosomes originating from MSCs stimulated with TGF-beta and IFN-gamma promote Treg differentiation. J Cell Physiol 2018;233:6832-40. https://doi.org/10.1002/jcp.26436.

[29] Can A, Celikkan FT, Cinar O. Umbilical cord mesenchymal stromal cell transplantations: A systemic analysis of clinical trials. Cytotherapy 2017;19:1351-82. https://doi.org/10.1016/ j.jcyt.2017.08.004.

[30] Bailey AM, Mendicino M, Au P. An FDA perspective on preclinical development of cell-based regenerative medicine products. Nat Biotechnol 2014;32:721-3. https://doi.org/ 10.1038/nbt.2971.

[31] Heinemann ML, Ilmer M, Silva LP, Hawke DH, Recio A, Vorontsova MA, et al. Benchtop isolation and characterization of functional exosomes by sequential filtration. J Chromatogr A 2014;1371:125-35. https://doi.org/10. 1016/j.chroma.2014.10.026.

[32] Gamez-Valero A, Monguio-Tortajada M, Carreras-Planella L, Franquesa M, Beyer K, Borras FE. Size-exclusion chromatography-based isolation minimally alters extracellular vesicles' characteristics compared to precipitating agents. Sci Rep 2016;6:33641. https://doi.org/10.1038/srep33641.

[33] European Medicines Agency. Guideline on the requirements for quality documentation concerning biological investigational medicinal products in clinical trials. http://www.ema. europa.eu/ema/index.jsp?curl=pages/regulation/general/ general_content_000951.jsp\&mid=WC0b01ac058002956c. As by August 8, 2018.

[34 International Council for Harmonisation. ICHQ6B. Test Procedures and Acceptance Criteria for Biotechnological/Biological Products.

[35] Dominici M, Le Blanc K, Mueller I, Slaper-Cortenbach I, Marini F, Krause D, et al. Minimal criteria for defining multipotent mesenchymal stromal cells. The International Society for Cellular Therapy position statement. Cytotherapy 2006;8:315-7.

[36] Webber J. Clayton A How pure are your vesicles. J Extracell Vesicles 2013;2. https://doi.org/10.3402/jev.v2i0.19861.

[37] Wiklander OPB, Bostancioglu RB, Welsh JA, Zickler AM, Murke F, Corso G, et al. Systematic Methodological Evaluation of a Multiplex Bead-Based Flow Cytometry Assay for Detection of Extracellular Vesicle Surface Signatures. Frontiers in immunology 2018;9:1326. https://doi.org/10.3389/ fimmu.2018.01326.

[38] Fatima F, Ekstrom K, Nazarenko I, Maugeri M, Valadi H, Hill AF, et al. Non-coding RNAs in mesenchymal stem cellderived extracellular vesicles: deciphering regulatory roles in stem cell potency, inflammatory resolve, and tissue regeneration. Front Genet 2017;8:161. https://doi.org/10.3389/ fgene.2017.00161.

[39] Qian X, Xu C, Fang S, Zhao P, Wang Y, Liu H, et al. Exosomal MicroRNAs Derived From Umbilical Mesenchymal Stem Cells Inhibit Hepatitis C Virus Infection. Stem Cells Transl Med 2016;5:1190-203. https://doi.org/10.5966/ sctm.2015-0348.

[40] Sharif S, Ghahremani MH, Soleimani M. Delivery of Exogenous miR-124 to Glioblastoma multiform cells by Wharton's jelly mesenchymal stem cells decreases cell proliferation and migration, and confers chemosensitivity. Stem Cell Rev 2018;14:236-46. https://doi.org/10.1007/ s12015-017-9788-3. 
[41] Dong L, Pu Y, Zhang L, Qi Q, Xu L, Li W, et al. Human umbilical cord mesenchymal stem cell-derived extracellular vesicles promote lung adenocarcinoma growth by transferring miR-410. Cell death $\&$ disease 2018;9:218. https://doi. org/10.1038/s41419-018-0323-5.

[42] U.S. Food and Drug Administration. 21CFR11, Code of Federal Regulations Title 21. Volume 1; https://www.accessdata.fda.gov/scripts/cdrh/cfdocs/cfCFR/CFRSearch.cfm? CFRPart $=11$ \&showFR=1. Retrieved August 8, 2018.

[43] Colao IL, Corteling R, Bracewell D, Wall I. Manufacturing exosomes: a promising therapeutic platform. Trends $\mathrm{Mol}$ Med 2018;24:242-56. https://doi.org/10.1016/j.molmed.2018.01.006.

[44] Parisse P, Rago I, Ulloa Severino L, Perissinotto F, Ambrosetti E, Paoletti P, et al. Atomic force microscopy analysis of extracellular vesicles. Eur Biophys J 2017;46:813-20. https:// doi.org/10.1007/s00249-017-1252-4.

[45] Escudier B, Dorval T, Chaput N, Andre F, Caby MP, Novault $S$, et al. Vaccination of metastatic melanoma patients with autologous dendritic cell (DC) derived-exosomes: results of thefirst phase I clinical trial. J Transl Med 2005;3:10. https://doi.org/10.1186/1479-5876-3-10.

[46] Morse MA, Garst J, Osada T, Khan S, Hobeika A, Clay TM, et al. A phase I study of dexosome immunotherapy in patients with advanced non-small cell lung cancer. J Transl Med 2005;3:9. https://doi.org/10.1186/1479-5876-3-9.

[47] Besse B, Charrier M, Lapierre V, Dansin E, Lantz O, Planchard D, et al. Dendritic cell-derived exosomes as maintenance immunotherapy after first line chemotherapy in
NSCLC. Oncoimmunology 2016;5:e1071008. https://doi. org/10.1080/2162402X.2015.1071008.

[48] Suzuki E, Fujita D, Takahashi M, Oba S, Nishimatsu H. Stem cell-derived exosomes as a therapeutic tool for cardiovascular disease. World J Stem Cells 2016;8:297-305. https://doi.org/10.4252/wjsc.v8.i9.297.

[49] Dai S, Wei D, Wu Z, Zhou X, Wei X, Huang H, et al. Phase I clinical trial of autologous ascites-derived exosomes combined with GM-CSF for colorectal cancer. Mol Ther 2008;16:782-90. https://doi.org/10.1038/mt.2008.1.

[50] Ji R, Zhang B, Zhang X, Xue J, Yuan X, Yan Y, et al. Exosomes derived from human mesenchymal stem cells confer drug resistance in gastric cancer. Cell cycle (Georgetown, Tex.) 2015;14:2473-83. https://doi.org/ 10.1080/15384101.2015.1005530.

[51] Gu H, Ji R, Zhang X, Wang M, Zhu W, Qian H, et al. Exosomes derived from human mesenchymal stem cells promote gastric cancer cell growth and migration via the activation of the Akt pathway. Molecular medicine reports 2016;14:34528. https://doi.org/10.3892/mmr.2016.5625.

[52] Mendt M, Kamerkar S, Sugimoto H, McAndrews KM, Wu $\mathrm{CC}, \mathrm{Gagea} \mathrm{M}$, et al. Generation and testing of clinical-grade exosomes for pancreatic cancer. JCI insight 2018;3. https:// doi.org/10.1172/jci.insight.99263.

[53] Kamerkar S, LeBleu VS, Sugimoto H, Yang S, Ruivo CF, Melo SA, et al. Exosomes facilitate therapeutic targeting of oncogenic KRAS in pancreatic cancer. Nature 2017;546:498-503. https://doi.org/10.1038/nature22341. 